\title{
Correlación entre niveles de ácido úrico y velocidad de la onda del pulso en sujetos con síndrome metabólico
}

\author{
Alberto F. Rubio-Guerra ${ }^{1 *}$, Jorge L. Narváez-Rivera², Daniel R. Benítez-Maldonado², \\ Carolina Guerrero-García ${ }^{1}$, Karla C. Arana-Pazos ${ }^{1}$ y Germán Vargas-Ayala ${ }^{3}$ \\ ${ }^{1}$ Servicio de Enseñanza e Investigación; ${ }^{2}$ Servicios de Ecocardiografía; ${ }^{3}$ Servicio de Medicina Interna. Hospital General de Ticomán, Secretaría de \\ Salud, Ciudad de México, México
}

\section{Resumen}

Introducción: La rigidez de la pared arterial es un predictor independiente de mortalidad cardiovascular. Se evalúa midiendo la velocidad de la onda del pulso (VOP), un procedimiento no invasivo y validado. El exceso de ácido úrico genera disfunción endotelial y daño vascular, lo que favorece la fibrosis y remodelación vascular, y con ello rigidez arterial. Objetivo: Evaluar si hay correlación entre los niveles de ácido úrico y la VOP en sujetos con síndrome metabólico. Método: Se evaluaron 165 pacientes con síndrome metabólico, en quienes se determinó la VOP usando un equipo Arteriograph. Se consideró normal una VOP $\leq 9 \mathrm{~m} / \mathrm{s}$. En todos ellos se realizó la determinación de ácido úrico (método enzimático). Los métodos estadísticos usados fueron coeficiente de correlación de Pearson y razón de momios. Resultados: Encontramos una correlación significativa $\left(r=0.41, r^{2}=0.16 ; p<0.00001\right)$ entre la VOP y los niveles séricos de ácido úrico, la razón de momios para presentar VOP aumentada en sujetos con hiperuricemia fue de 5.62 (IC 95\%: 1.92-16.45). Conclusión: Nuestros resultados sugieren que el ácido úrico tiene un papel sobre los mecanismos que llevan a la rigidez arterial. Estudios posteriores determinarán si disminuir sus niveles séricos mejorarían la rigidez arterial.

Palabras clave: Ácido úrico. Velocidad de la onda del pulso. Rigidez arterial. Daño vascular.

\section{Correlation between uric acid levels and pulse wave velocity in patients with metabolic syndrome}

\section{Abstract}

Introduction: Arterial stiffness is an independent marker of cardiovascular mortality, pulse wave velocity (PWV) is the standard for the measurement of arterial stiffness, it is a non-invasive and validated procedure. Hyperuricemia produces endothelial dysfunction and vascular damage, and may lead to fibrosis, vascular remodelling and arterial stiffness. Objective: To evaluate if there is correlation between uric acid levels and PWV in subjects with metabolic syndrome. Method: 165 patients with metabolic syndrome were included, in whom PWV was measured using an Arteriograph equip A PWV $\leq 9 \mathrm{~m} / \mathrm{s}$ was considered normal. In all of them uric acid was measured (enzymatic method) and statistical analysis was performed with Pearson correlation coefficient and odds ratio. Results: We found a positive correlation $\left(r=0.41, r^{2}=0.16 ; p<0.00001\right)$ between uric acid levels and PWV. The odds ratio for an increase in PWV in patients with hyperuricemia was 5.62 (95\% confidence interval: 1.92-16.45). Conclusion: Our results suggest a role of uric acid in the pathways that lead to arterial stiffness. Future studies will determine if treatment for uricemia may improve arterial stiffness.

Key words: Uric acid. Pulse wave velocity. Arterial stiffness. Vascular damage.

\footnotetext{
Correspondencia:

${ }^{\star}$ Alberto F. Rubio-Guerra

Fecha de recepción: 18-01-2019

Fecha de aceptación: 08-11-2019

DOI: 10.24875/NEFRO.19000058

E-mail: clinhta@hotmail.com 2444-9032/@ 2019 Sociedad Latinoamericana de Nefrología e Hipertensión. Publicado por Permanyer. Éste es un artículo open access bajo la licencia CC BY-NC-ND (http://creativecommons.org/licenses/by-nc-nd/4.0/).

Disponible en internet: 18-12-2019 Nefro Latinoam. 2019;16:33-38 www.nefrologialatinoamericana.com
} 


\section{Introducción}

La rigidez arterial es producto de la remodelación de las grandes arterias, habitualmente como un mecanismo compensatorio a la hipertensión crónica. Es un proceso en el que además de la hipertensión arterial participan el envejecimiento, la dislipidemia, la edad y el sexo, entre otros factores, y en el que ocurren cambios en la composición y propiedades físicas de las proteínas estructurales de la pared arterial. Dentro de los mecanismos involucrados en su desarrollo se encuentra la actividad del eje renina-angiotensina-aldosterona, y la angiotensina II es un promotor importante de la disfunción endotelial, inflamación y fibrosis vascular, activación de factores de crecimiento y migración de células musculares que aumentan el depósito de la matriz extracelular. La rigidez arterial se asocia a un aumento del riesgo cardiovascular ${ }^{1}$.

La rigidez arterial se puede medir clínicamente determinando la velocidad de la onda del pulso (VOP), un procedimiento no invasivo, seguro y validado. Cuando la VOP se encuentra aumentada, se considera también como un marcador de riesgo cardiovascular ${ }^{2}$. Se considera normal una VOP entre 6.1 y $9.1 \mathrm{~m} / \mathrm{s}$, dependiendo de la edad, el sexo y la presión arterial ${ }^{3,4}$.

El ácido úrico es el producto final de la degradación de las purinas en los primates, incluidos el humano, la hiperuricemia puede ocasionar disfunción endotelial y daño arterial por diversos mecanismos, tales como activación del eje renina-angiotensina, disminución en la biodisponibilidad del óxido nítrico, generación de resistencia a la insulina y disminución en la liberación de adiponectina ${ }^{5}$.

Las concentraciones de ácido úrico se correlacionan con el grosor íntima-media, lo que sugiere la participación del ácido úrico en el proceso de aterosclerosis, por ello, el aumento en los niveles de ácido úrico se ha asociado a un aumento del riesgo cardiovascular ${ }^{6}$.

La hiperuricemia también produce proliferación del músculo liso vascular y cambios en las propiedades vasculares, aunque su asociación con la rigidez arterial ha mostrado resultados contradictorios?

El objetivo de este estudio es evaluar si existe correlación entre la VOP y los niveles séricos de ácido úrico y otros marcadores bioquímicos de riesgo cardiovascular.

\section{Material y métodos}

Se evaluaron 165 pacientes con síndrome metabólico provenientes de la consulta externa del hospital, en quienes se determinó la VOP usando un equipo
Arteriograph, que es un instrumento validado ${ }^{8}$, y algunos autores lo han propuesto como el estándar de oro para medir la $\mathrm{VOP}^{3}$. Se colocó un brazalete a nivel braquial, conectado a un sensor, se infló $35 \mathrm{mmHg}$ por encima de la presión sistólica para ocluir completamente la arteria, y se midieron dos picos de presión, el primero es resultado de la eyección ventricular hacia la aorta y el segundo mide la onda de reflección, y se determinó el intervalo de tiempo entre ambos picos. Se consideró normal una VOP $\leq 9 \mathrm{~m} / \mathrm{s}$.

Así mismo, en todos ellos se realizó de forma inmediata las determinaciones de glucosa sérica (glucosa oxidasa), creatinina sérica (Jaffe), ácido úrico, perfil de lípidos con colesterol (CHOD-PAP) y triglicéridos (triglicérido-PAP) -la cuantificación de LDL se realizó mediante el método de Friedewald en quienes presentaron valores de triglicéridos $<400 \mathrm{mg} / \mathrm{dl}$, y en quienes presentaron valores superiores a estos, se determinaron directamente- por personal que desconocía el grupo al que pertenecían los pacientes. Se utilizó un equipo UniCel DXC 600 (Beckman Coulter Eurocenter [Nyon, Suiza]), con reactivos fabricados por la misma casa.

El ácido úrico se cuantificó por método de punto final a tiempo con ReactivoURIC.

Todas las muestras se tomaron por punción venosa (aproximadamente $15 \mathrm{ml}$ ), después de un ayuno de 8 horas, en tubos sin anticoagulante.

Se consideró hiperuricemia valores de ácido úrico > $6.5 \mathrm{mg} / \mathrm{dl}$ en el varón y $>5.1 \mathrm{mg} / \mathrm{dl}$ en la mujer ${ }^{5}$. El diagnóstico de síndrome metabólico se hizo con los criterios de la Federación Internacional de Diabetes ${ }^{9}$.

Se excluyeron pacientes con alguno de los siguientes diagnósticos: insuficiencia cardíaca, hepática (aminotransferasas séricas aumentadas a más del doble de su límite superior normal) y/o renal (creatinina > $2.5 \mathrm{mg} / \mathrm{dl}$ ), diabetes mellitus descompensada (glucemia $>250 \mathrm{mg} / \mathrm{dl}$ ), asimismo pacientes con historia de abuso de alcohol o drogas psicotrópicas, antecedente de ingesta de tiazidas, alopurinol o febuxostat los últimos 6 meses, 0 antecedentes de quimioterapia antineoplásica en el último año.

\section{Análisis estadístico}

Los métodos estadísticos usados fueron el coeficiente de correlación de Pearson, $U$ de Mann-Withney y razón de momios. Los datos se presentan como media \pm desviación estándar, se consideró significativa una $p<0.05$. 
Tabla 1. Características de los pacientes

\begin{tabular}{|c|c|c|c|c|}
\hline & $\begin{array}{l}\text { Hiperuricemia } \\
\text { (96 pacientes) }\end{array}$ & $\begin{array}{l}\text { Normouricemia } \\
\text { (69 pacientes) }\end{array}$ & $\begin{array}{l}\text { p (entre los } \\
\text { grupos) }\end{array}$ & Total de pacientes \\
\hline Edad (años) & $52.9 \pm 13$ & 53.8. \pm 12.6 & 0.380 & $53.1 \pm 7$ \\
\hline Sexo (M/F) & $32 / 64$ & $23 / 46$ & & $55 / 110$ \\
\hline Frecuencia cardíaca (latidos por minuto) & $71 \pm 7.6$ & $72 \pm 7.5$ & 0.37 & $71 \pm 7.3$ \\
\hline Índice de masa corporal $\left(\mathrm{Kg} / \mathrm{m}^{2}\right)$ & $30.8 \pm 4.3$ & $28.4 \pm 5$ & 0.005 & $29.4 \pm 3.9$ \\
\hline Perímetro abdominal $(\mathrm{cm})$ & $98.3 \pm 10.5$ & $93.8 \pm 11.4$ & 0.020 & $97.9 \pm 9$ \\
\hline Historia de hipertensión arterial (\%) & $43(44.8 \%)$ & $18(26 \%)$ & & 61 \\
\hline Historia de diabetes mellitus tipo $2(\%)$ & $32(33 \%)$ & $22(31.9 \%)$ & & 54 \\
\hline Tabaquismo (\%) & $28(29 \%)$ & $19(27.5 \%)$ & & 47 \\
\hline Ingesta de estatinas (\%) & $28(29.1 \%)$ & $20(28.9 \%)$ & & 48 \\
\hline Presión arterial $(\mathrm{mmHg})$ & $128 \pm 14 / 82 \pm 11$ & $128 \pm 16 / 78 \pm 10$ & 0.900 & $128 \pm 14 / 81 \pm 10$ \\
\hline Glucemia (mg/dl) & $123.7 \pm 37$ & $122 \pm 45$ & 0.700 & $123 \pm 39$ \\
\hline Triglicéridos (mg/dl) & $223 \pm 93$ & $206 \pm 92$ & 0.380 & $219.9 \pm 32$ \\
\hline $\begin{array}{l}\text { Colesterol transportado por lipoproteínas de } \\
\text { baja densidad (mg/dl) }\end{array}$ & $122 \pm 30$ & $119 \pm 29$ & 0.88 & $121 \pm 24$ \\
\hline $\begin{array}{l}\text { Colesterol transportado por lipoproteínas de } \\
\text { alta densidad }(\mathrm{mg} / \mathrm{dl})\end{array}$ & $42 \pm 11$ & $44 \pm 9$ & 0.150 & $40.9 \pm 8.8$ \\
\hline Ácido úrico (mg/dl) & $7.2 \pm 1.6$ & $4.7 \pm 0.8$ & 0.00001 & $5.8 \pm 1.34$ \\
\hline
\end{tabular}

\section{Aspectos éticos}

El estudio fue aprobado por el Comité de enseñanza, investigación, capacitación y ética del Hospital General de Ticomán SS DF, con registro 208/010/19/16. Se realizó de acuerdo con la declaración de Helsinki, los pacientes dieron su consentimiento informado antes de ser incluidos.

\section{Resultados}

Las características de los pacientes se muestran en la tabla 1.

El manejo antihipertensivo de los pacientes se muestra en la tabla 2.

Los pacientes con hiperuricemia presentaron una VOP significativamente mayor que los sujetos normouricémicos (9.83 \pm 1.5 vs. $8.94 \pm 1.2 ; p=0.037$ ).

No encontramos correlación de la VOP con el índice de masa corporal $(r=0.023 ; p=0.76)$, perímetro abdominal $(r=0.11 ; p=0.16)$, glucemia $(r=0.06 ; p=0.7)$, triglicéridos $(r=0.1 ; p=0.56)$, lipoproteínas de baja densidad $(r=0.11 ; p=0.52)$, ni lipoproteínas de alta densidad $(r=-0.01 ; p=0.9)$.
Tabla 2. Manejo antihipertensivo de los pacientes

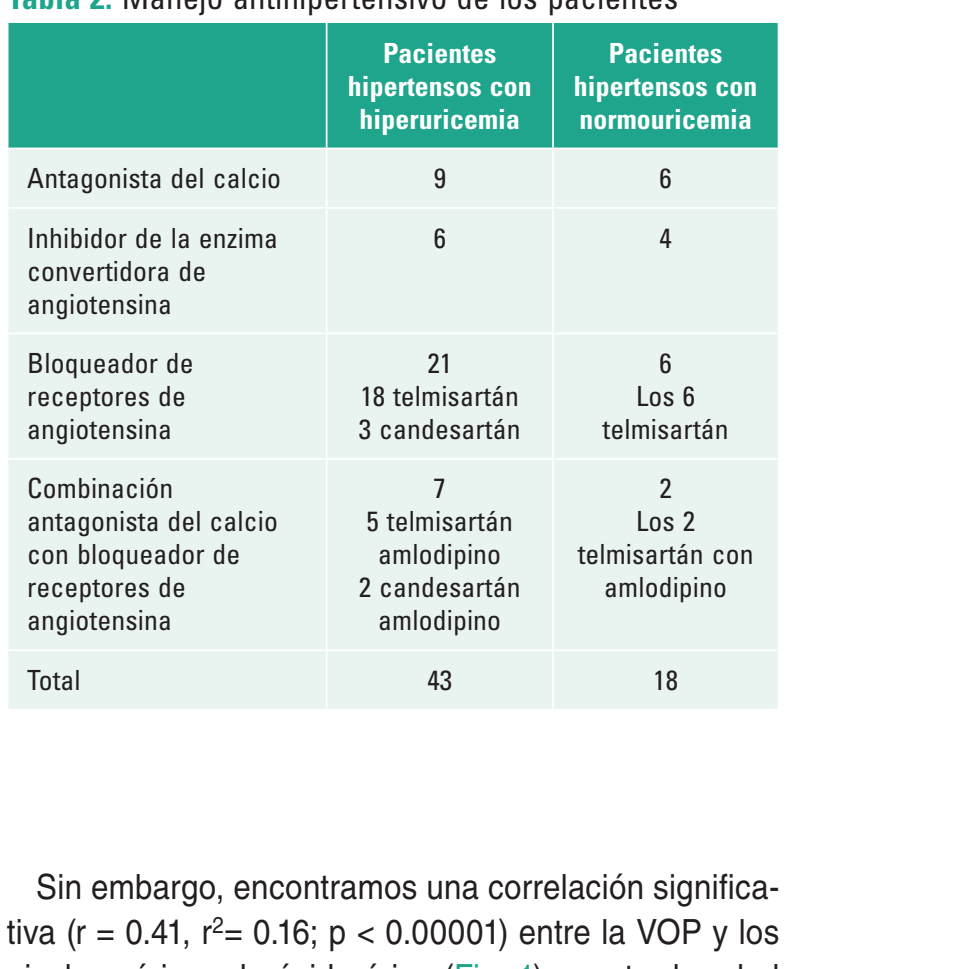
niveles séricos de ácido úrico (Fig. 1), y entre la edad y la VOP $(r=0.30 ; p=0.0009)$. 


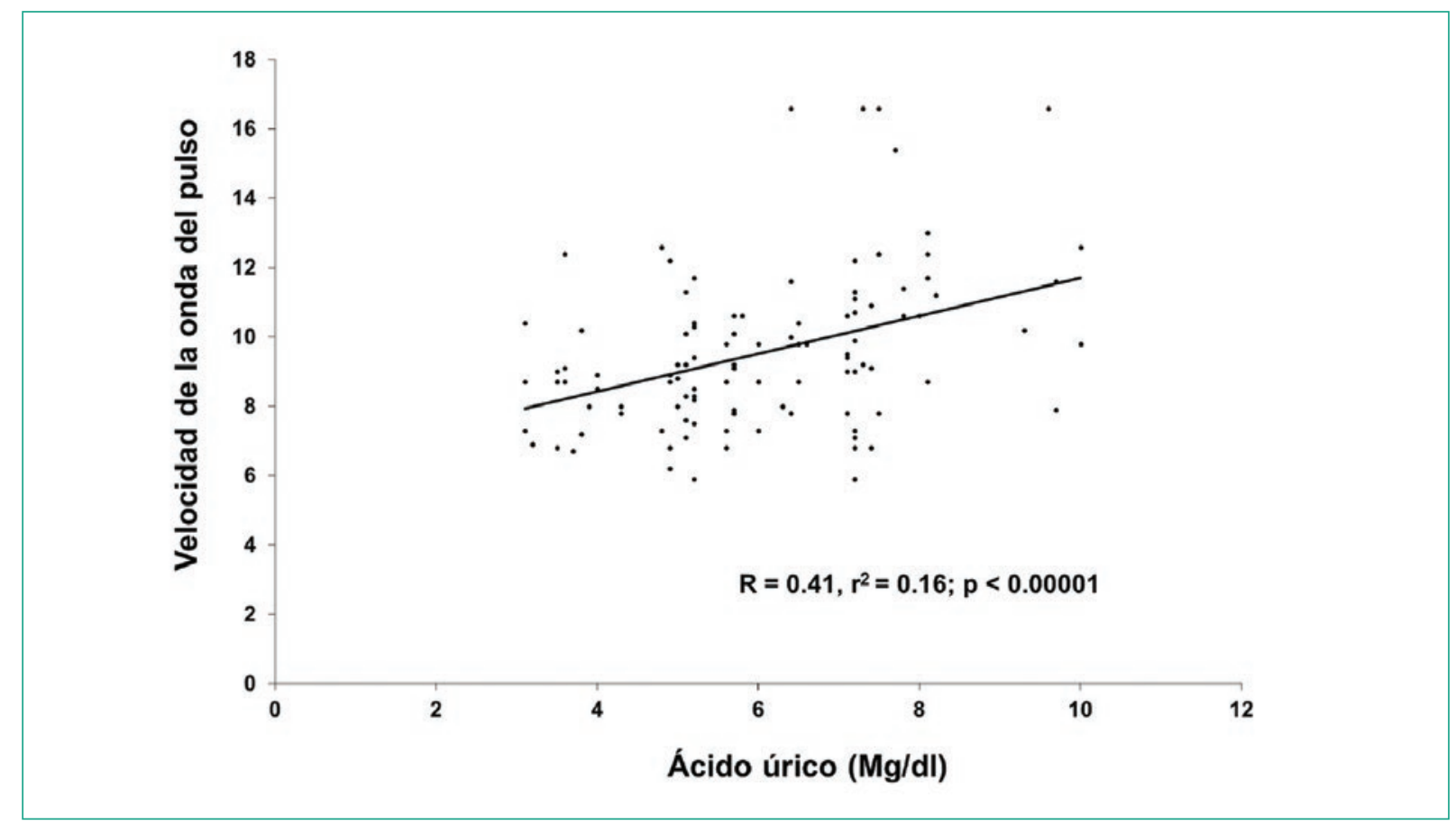

Figura 1. Correlación entre la velocidad de la onda de pulso y los niveles séricos de ácido úrico.

La razón de momios para presentar una VOP aumentada en sujetos con hiperuricemia fue de 5.62 (IC 95\%: 1.92-16.45).

Al realizar un análisis de regresión logística múltiple, encontramos que el ácido úrico se asoció a la VOP de manera independiente a las demás variables (Tabla 3).

\section{Discusión}

En este estudio encontramos correlación entre la VOP y los niveles séricos de ácido úrico, así como con la edad. Esta correlación se da en forma independiente a otros marcadores clínicos y bioquímicos de riesgo cardiovascular, tales como la glucemia, los valores séricos de lipoproteínas y los triglicéridos. La razón de momios encontrada nos muestra que la presencia de hiperuricemia aumenta el riesgo de rigidez arterial.

El ácido úrico altera la distensibilidad arterial por sus efectos sobre la disfunción endotelial y el óxido nítrico. La hiperuricemia se ha identificado como un predictor de la aparición y progresión de la hipertensión arterial, aunado a ello, la asociación de hiperuricemia con daño vascular se conoce desde hace tiempo, la hiperuricemia se ha asociado a inflamación arterial, proliferación de células musculares lisas en los vasos, interferencia con el funcionamiento de barorreflejos y desarrollo de aterosclerosis ${ }^{6}$. Además, la activación del eje renina
Tabla 3. Análisis de regresión logística múltiple de la relación entre velocidad de la onda del pulso y otros marcadores de riesgo metabólico

\begin{tabular}{|l|c|c|}
\hline Variable & B & p \\
\hline Edad & 0.27 & 0.0009 \\
\hline Ácido úrico & 0.39 & 0.0001 \\
\hline Índice de masa corporal & 0.009 & 0.76 \\
\hline Perímetro abdominal & 0.002 & 0.16 \\
\hline Glucemia & 0.009 & 0.700 \\
\hline Lipoproteínas de baja densidad & 0.005 & 0.520 \\
\hline Triglicéridos & 0.005 & 0.560 \\
\hline $\begin{array}{l}\text { Colesterol transportado por lipoproteínas } \\
\text { de alta densidad }\end{array}$ & -0.0001 & 0.900 \\
\hline
\end{tabular}

angiotensina inducida por el ácido úrico favorece la remodelación vascular y el depósito de colágeno en la pared arterial ${ }^{1,5}$.

A su vez, el incremento en la rigidez arterial favorece la hipertrofia del ventrículo izquierdo, aumenta el trabajo ventricular y reduce el flujo coronario. Por ello, la rigidez arterial se asocia con mayor riesgo de eventos coronarios. De hecho, la Sociedad Europea de Cardiología recomienda la medición de la VOP para evaluar 
el daño subclínico a órganos blanco. La rigidez arterial también participa en el desarrollo de hipertensión arterial $^{10}$.

Cicero, et al. encontraron que los niveles séricos de ácido úrico predicen la VOP en sujetos con función renal conservada, pero no cuando la función renal está más comprometida ${ }^{11}$, es importante señalar que, en nuestro estudio, todos los pacientes cursaban con niveles de creatinina $<2.5 \mathrm{mg} / \mathrm{dl}$; mientras que Mulè, et al. encontraron asociación entre hiperuricemia y rigidez arterial en pacientes hipertensos no tratados y con hiperuricemia leve ${ }^{12}$; sin embargo, la asociación que ellos encontraron fue independiente de la presencia de síndrome metabólico. A su vez, Cicero, et al. ${ }^{13}$ encontraron asociación entre niveles séricos de ácido úrico con rigidez arterial en hipertensos mal controlados con fármacos antihipertensivos.

Vlachopoulos, et al. encontraron asociación entre la rigidez aórtica y el índice de aumento en sujetos hipertensos recién diagnosticados y sin tratamiento antihipertensivo ${ }^{14}$.

En nuestro estudio incluimos población con síndrome metabólico, pero a diferencia de los estudios comentados previamente, participaron sujetos normouricémicos y normotensos. Resulta interesante la correlación de la VOP con la uricemia, incluso al haber pacientes con valores normales de ácido úrico, aunque ciertamente el número de sujetos hipertensos fue mayor en el grupo con hiperuricemia.

Además del proceso de inflamación vascular y disfunción endotelial, se han reportado otros mecanismos que explicarían la correlación entre los valores séricos de ácido úrico y la VOP que encontramos en este estudio. Gruszka, et al. encontraron una asociación fuerte entre los niveles séricos de ácido úrico y la actividad de la enzima matriz metaloproteinasa 3 , la cual tiene un papel fundamental en la activación de otras metaloproteasas. El resultado final es la remodelación vascular y formación de placas de ateroma ${ }^{15}$. Es interesante señalar que Tsai, et al. reportaron que la asociación entre ácido úrico y VOP no es secundaria a un aumento del proceso inflamatorio ${ }^{16}$.

El síndrome metabólico, además de ser un factor independiente de riesgo cardiovascular, acelera el envejecimiento vascular y favorece la rigidez arterial, y se ha descrito que los valores de triglicéridos y lipoproteínas de alta densidad se asocian a un aumento de la rigidez arterial ${ }^{17}$. En nuestro estudio no hubo diferencias en los valores séricos de ambos lípidos en ambos grupos, ni correlación entre ellos y la VOP.
Como limitantes a nuestro estudio, cabe señalar que se trata de un estudio transversal que muestra asociación, no causalidad, y el reducido tamaño de la muestra.

Como conclusión encontramos que la hiperuricemia se asocia a rigidez arterial en pacientes con síndrome metabólico, con una correlación (débil) entre los niveles séricos de ácido úrico y la VOP en estos pacientes.

\section{Conflicto de intereses}

Los autores declaran que no existe conflicto de intereses.

\section{Responsabilidades éticas}

Protección de personas y animales. Los autores declaran que los procedimientos seguidos se conformaron a las normas éticas del comité de experimentación humana responsable y de acuerdo con la Asociación Médica Mundial y la Declaración de Helsinki.

Confidencialidad de los datos. Los autores declaran que han seguido los protocolos de su centro de trabajo sobre la publicación de datos de pacientes.

Derecho a la privacidad y consentimiento informado. Los autores han obtenido el consentimiento informado de los pacientes y/o sujetos referidos en el artículo. Este documento obra en poder del autor de correspondencia.

\section{Bibliografía}

1. Rubio-Guerra AF, Duran-Salgado MB. Antihypertensive treatment and vascular extracellular matrix remodeling. Cardiology. 2014;127:245-6.

2. Ohkuma T, Ninomiya T, Tomiyama H, Kario K, Hoshide S, Kita Y, et al. Collaborative Group for J-BAVEL (Japan Brachial-Ankle Pulse Wave Velocity Individual Participant Data Meta-Analysis of Prospective Studies) ${ }^{\star}$. Brachial-Ankle Pulse Wave Velocity and the Risk Prediction of Cardiovascular Disease: An Individual Participant Data Meta-Analysis. Hypertension. 2017:69:1045-52.

3. Davies JM, Bailey MA, Griffin KJ, Scott DJ. Pulse wave velocity and the non-invasive methods used to assess it: Complior, SphygmoCor, Arteriograph and Vicorder. Vascular. 2012;20:342-9.

4. Reference Values for Arterial Stiffness' Collaboration. Determinants of pulse wave velocity in healthy people and in the presence of cardiovascular risk factors: 'establishing normal and reference values'. Eur Heart J. 2010;31:2338-50

5. Rubio-Guerra AF, Morales-López H, Garro-Almendaro AK, Vargas-Ayala G, Durán-Salgado MB, Huerta-Ramírez S, et al. Circulating Levels of Uric Acid and Risk for Metabolic Syndrome. Curr Diabetes Rev. 2017;13:87-90.

6. Tavil Y, Kaya MG, Oktar SO, Sen N, Okyay K, Yazici HU, et al. Uric acid level and its association with carotid intima-media thickness in patients with hypertension. Atherosclerosis. 2008;197:159-63.

7. Bae JS, Shin DH, Park PS, Choi BY, Kim MK, Shin MH, et al. The impact of serum uric acid level on arterial stiffness and carotid atherosclerosis: the Korean Multi-Rural Communities Cohort study. Atherosclerosis. 2013; 231:145-51.

8. Ring M, Eriksson MJ, Zierath JR, Caidahl K. Arterial stiffness estimation in healthy subjects: a validation of oscillometric (Arteriograph) and tonometric (SphygmoCor) techniques. Hypertens Res. 2014;37:999-1007. 
9. Alberti KG, Eckel RH, Grundy SM, Zimmet PZ, Cleeman JI, Donato KA et al.; International Diabetes Federation Task Force on Epidemiology and Prevention; National Heart, Lung, and Blood Institute; American Heart Association; World Heart Federation; International Atherosclerosis Society; International Association for the Study of Obesity. Harmonizing the metabolic syndrome: a joint interim statement of the International Diabetes Federation Task Force on Epidemiology and Prevention; National Heart, Lung, and Blood Institute; American Heart Association; World Heart Federation; International Atherosclerosis Society; and International Association for the Study of Obesity. Circulation. 2009;120:1640-5.

10. Vlachopoulos C, Aznaouridis K, Stefanadis C. Prediction of cardiovascular events and all-cause mortality with arterial stiffness: a systematic review and meta-analysis. J Am Coll Cardiol. 2010;55:1318-27.

11. Cicero AFG, Kuwabara M, Johnson R, Bove M, Fogacci F, Rosticci M, Giovannini M, D'Addato S, Borghi C; Brisighella Heart Study group. LDL-oxidation, serum uric acid, kidney function and pulse-wave velocity: Data from the Brisighella Heart Study cohort. Int J Cardiol. 2018;261:204-8.

12. Mulé $G$, Riccobene $R$, Castiglia A, D'Ignoto F, Ajello E, Geraci $G$, et al Relationships between mild hyperuricaemia and aortic stiffness in untreated hypertensive patients. Nutr Metab Cardiovasc Dis. 2014;24:744-50.
13. Cicero AF, Rosticci M, Fogacci F, Grandi E, D'Addato S, Borghi C; Brisighella Heart Study Group. High serum uric acid is associated to poorly controlled blood pressure and higher arterial stiffness in hypertensive subjects. Eur J Intern Med. 2017;37:38-42.

14. Vlachopoulos C, Xaplanteris P, Vyssoulis G, Bratsas A, Baou K, Tzamou $V$ y cols. Association of serum uric acid level with aortic stiffness and arterial wave reflections in newly diagnosed, never-treated hypertension. Am J Hypertens. 2011;24:33-9.

15. Gruszka K, Rajzer M, DrożdżT, WojciechowskaW, Pizo囚T, Migacz-Gruszka K, Czarnecka D. Selected matrix metalloproteinases activity and hypertension-mediated organ damage in relation to uric acid serum level. Cardiol $\mathrm{J}$. 2019 Apr 17. doi: 10.5603/CJ.a2019.0033. [Epub ahead of print].

16. Tsai WC, Huang YY, Lin CC, Li WT, Lee CH, Chen JY, Chen JH. Uric acid is an independent predictor of arterial stiffness in hypertensive patients. Heart Vessels. 2009:24:371-5.

17. Chen MC, Lee CJ, Yang CF, Chen YC, Wang JH, Hsu BG. Low serum adiponectin level is associated with metabolic syndrome and is an independent marker of peripheral arterial stiffness in hypertensive patients. Diabetol Metab Syndr. 2017;9:49-58. 